\title{
Efetividade do treinamento em habilidades sociais na qualidade de vida de trabalhadores: uma estratégia de promoção da saúde
}

\author{
Effectiveness of social skills training on workers quality of life: a health promotion strategy \\ Efectividad de la capacitación en habilidades sociales sobre la calidad de vida de los \\ trabajadores: una estrategia de promoción de la salud
}

Patricia Ramos Branco ${ }^{1}$, Elisabete Agrela de Andrade ${ }^{1 *}$.

\section{RESUMO}

Objetivo: Avaliar a efetividade de um treinamento em habilidades sociais na melhoria da qualidade de vida de um grupo de trabalhadores. Métodos: De abordagem quantitativa, em uma instituição de ensino superior na cidade de São Paulo, foi realizada uma pesquisa de avaliação pré e pós-intervenção de um treinamento de habilidades sociais, com dez sessões, em dez profissionais de atendimento ao cliente. Foram aplicados questionário sociodemográfico; Inventário de Habilidades Sociais (IHS-Del-Prette) e a versão brasileira do questionário de qualidade de vida SF-36., antes e depois do treinamento. Os dados obtidos foram analisados estatisticamente com os testes Wilcoxon e Spearman para correlacionar os dois instrumentos IHS-Del-Prette e SF. A pesquisa foi aprovada pelo Comitê de Ética em Pesquisa. Resultados: O grupo foi composto por $80 \%$ de mulheres, com média de idade de 27,5 anos, $80 \%$ com nível superior completo. O treinamento em habilidades sociais se mostrou efetivo na ampliação do repertório de habilidades sociais, comparando os resultados fase pré-teste e pós-teste, assim como mostrou que há correlação positiva entre habilidades sociais e melhoria da qualidade de vida. Conclusão: $O$ treinamento em habilidades sociais pode ser considerada uma estratégia efetiva de promoção à saúde, pois repercute positivamente para a construção de ambiente saudável, para os relacionamentos, melhorando o bem estar físico, mental e social, contribuindo então a qualidade de vida dos trabalhadores.

Palavras-chave: Habilidades sociais, Promoção da saúde, Saúde do trabalhador.

\begin{abstract}
Objective: To evaluate the effectiveness of training in social skills in improving the quality of life of a group of workers. Methods: With a quantitative approach, a pre-and post-intervention evaluation of a social skills training was carried out, with ten sessions, in ten customer service professionals. Sociodemographic questionnaire was applied; Social Skills Inventory and SF-36 questionnaire, before and after training. The data obtained were analyzed statistically with the Wilcoxon and Spearman tests to correlate the two IHS-Del-Prette and SF instruments. The research was approved by the Research Ethics Committee. Results: The group was composed of $80 \%$ women, with an average age of 27.5 years, $80 \%$ with a college degree. Social skills training was effective in expanding the repertoire of social skills, comparing the pre-test and post-test results, as well as showing that there is a positive correlation between social skills and improved quality of life. Conclusion: It is concluded that training in social skills can be considered an effective health promotion strategy, as it has a positive impact on the construction of a healthy environment, for relationships, improving physical, mental and social well-being, thus contributing to quality workers' lives.
\end{abstract}

Key words: Social skills, Health promotion, Occupational health.

\section{RESUMEN}

Objetivo: Evaluar la efectividad de la capacitación en habilidades sociales para mejorar la calidad de vida de un grupo de trabajadores. Métodos: Con un enfoque cuantitativo, se realizó una evaluación previa y posterior a la intervención de una capacitación en habilidades sociales, con diez sesiones, en diez profesionales de servicio al cliente. Se aplicó cuestionario sociodemográfico; inventario de habilidades sociales y cuestionario SF-36, antes y después del entrenamiento. Los datos obtenidos se analizaron estadísticamente con las pruebas de Wilcoxon y Spearman para correlacionar los dos instrumentos IHS-Del-Prette y SF. La investigación fue aprobada por el Comité de Ética de Investigación. Resultados: El grupo estaba compuesto

${ }^{1}$ Centro Universitário Adventista de São Paulo (UNASP), São Paulo - SP.

*E-mail: elisabeteagrela1@gmail.com

SUBMETIDO EM: 5/2020 
por $80 \%$ de mujeres, con una edad promedio de 27.5 años, $80 \%$ con un título universitario. La capacitación en habilidades sociales fue efectiva para expandir el repertorio de habilidades sociales, comparando los resultados de la prueba previa y posterior a la prueba, y demostrando que existe una correlación positiva entre las habilidades sociales y la mejora de la calidad de vida. Conclusión: Se concluye que la capacitación en habilidades sociales puede considerarse una estrategia efectiva de promoción de la salud, ya que tiene un impacto positivo en la construcción de un entorno saludable, para las relaciones, la mejora del bienestar físico, mental y social, contribuyendo así a la calidad. la vida de los trabajadores.

Palabras clave: Habilidades sociales, Promoción de la salud, Salud laboral.

\section{INTRODUÇÃO}

Atualmente, cerca de $60 \%$ da população geral do Brasil está em idade ativa para o trabalho. Segundo o IBGE (2020), por outro lado, em 2010 a Organização Mundial da Saúde (OMS) aferiu que, a cada ano, cerca de dois milhões de pessoas morrem por acidentes, doenças ou lesões relacionadas ao trabalho, além disso, os riscos ocupacionais geram um ônus global de $8 \%$ causado por doenças oriundas da depressão.

Diante deste cenário, a OMS, baseada no referencial teórico da Promoção da Saúde, criou o Plano de Ação Global para fomentar ações de promoção da saúde dos trabalhadores incentivando a construção de ambientes de trabalho saudáveis. Há quatro áreas chave para tal construção: ambiente físico de trabalho; ambiente psicossocial de trabalho; recursos para a saúde pessoal; envolvimento da empresa na comunidade (OMS, 2010).

A promoção da saúde refere-se a um referencial e um modelo de atenção à saúde que vai além da assistência médico-curativa, onde a concepção de saúde é acatada como produção social, resultado de relações de distintas naturezas, quais sejam, econômicas, políticas, organizacionais, ideológicas, culturais e cognitivas. Dentro do escopo deste estudo, a promoção da saúde reconhece que decisões políticas de diferentes setores têm fortes repercussões sobre a saúde da população, aqui em especial, dos trabalhadores (ANDRADE EA e BOGUS CM, 2010).

A construção de ambiente psicossocial saudável deve levar em consideração a cultura organizacional, atitudes, crenças, valores e práticas de uma empresa que influenciam o bem-estar físico e mental de seus funcionários (OMS, 2010). O que está em consonância com o que a própria OMS chamou Ensino de Habilidades de Vida, que consiste no desenvolver capacidades emocionais, cognitivas e sociais que podem contribuir para que os sujeitos possam lidar com situações do cotidiano e também cooperar para a qualidade do ambiente em que vivem (WHO, 1997).

A literatura reforça a importância das habilidades sociais, na vida pessoal e profissional. Pois o repertório mais elaborado, os sujeitos estabelecem relações interpessoais e tendem a ter um fator de proteção, sendo menos vulneráveis a situação de risco a saúde (DEL PRETTE A e DEL PRETTE ZAP, 2003; MURTA SG,2005; RONDINA RC, 2010; CAMPOS JR, et al., 2018).

No ambiente de trabalho, onde existem muitas interações sociais, o cenário é propício para o desenvolvimento de problemas oriundos de relacionamentos entre as pessoas. $O$ excesso de relações conflituosas, estresse, altas demandas de produção são alguns destes fatores. Contribuir com o desenvolvimento humano, ofertar ferramentas para a vida profissional e pessoal, potencializar capacidades dos indivíduos são pontos importantes para gerar a promoção de saúde no trabalho e para o trabalho (SCHIRRMEISTER R e LIMONGI-FRANÇA C, 2012; SADIR MA e LIPP MN, 2013).

Ser socialmente habilidoso é conseguir ofertar respostas apropriadas às diferentes demandas sociais, além de possuir repertórios adequados à cultura que está imerso. Pessoas com repertório mais elaborado de habilidades sociais tendem a relatar maior satisfação com a vida porque percebem menos estresse; trabalham melhor; estudam melhor, tem suporte social e qualidade de vida, visto que lidam com as ansiedades, tristezas e frustrações de maneira mais apropriada e consequentemente, possuem estados cognitivos e afetivos mais positivos (CABALLO V, 2009; DEL PRETTE A e DEL PRETTE ZAP, 2001, 2010, 2011a; SEGRIN C e FLORA J, 2000). 
O Treinamento em Habilidades Sociais (THS) tem se apresentado como um procedimento relevante para ampliação do repertório de Habilidades Sociais (HS), ajudando na melhora da qualidade de vida dos sujeitos. Del Prette A e Del Prette ZAP (2011) conceituam as HS para o contexto de trabalho como aquelas que atendem às diferentes demandas interpessoais do ambiente de trabalho com objetivo de cumprir metas, preservar o bem-estar da equipe e respeitar os direitos de cada sujeito.

Através destes marcos conceituais, nota-se que o desenvolvimento de habilidades sociais para a construção de ambientes saudáveis é um importante instrumento para a qualidade de vida dos sujeitos. $O$ Treinamento em Habilidades Sociais pode ser uma ferramenta neste sentido, visto que contribui para que as pessoas consigam um melhor entendimento sobre suas forças pessoais no entendimento das relações sociais. Isto posto, ofertar instrumentos para a vida profissional e pessoal, potencializar capacidades nos indivíduos, são pontos importantes para gerar promoção de saúde para o trabalhador e para o ambiente de trabalho.

Entretanto, poucos estudos comprovam se o Treinamento em HS pode contribuir para a construção de ambientes saudáveis assim como para a qualidade de vida dos trabalhadores. Por isso o objetivo deste trabalho foi de avaliar a efetividade de um treinamento em habilidades sociais na melhoria da qualidade de vida de um grupo de trabalhadores.

\section{MÉTODOS}

Tratou-se de uma pesquisa de abordagem quantitativa. A avaliação da efetividade do treinamento foi realizada como um delineamento A-B (pré e pós-intervenção), sem grupo controle, o que o configura como um estudo pré-experimental. Ocorreu em uma instituição de ensino em São Paulo, com trabalhadores da área de atendimento ao cliente.

A amostragem foi não probabilística, a escolha dos elementos da amostra foi feita de forma não-aleatória, sendo intencional ou por conveniência, em que os sujeitos pertenciam ao quadro de funcionários do atendimento ao cliente de setores administrativos da instituição.

O convite foi destinado aos setores que tinham profissionais conforme o perfil de atendimento ao cliente: Participaram do treinamento 10 trabalhadores. O treinamento foi realizado na própria instituição, em sala apropriada. Os participantes tiveram no primeiro encontro todas as informações necessárias sobre o treinamento. Antes do início da pesquisa todos assinaram o termo de consentimento livre e esclarecido, onde são explicitados os objetivos do estudo, a garantia do sigilo em relação à identificação dos participantes, bem como a participação voluntária dos sujeitos.

No que diz respeito ao treinamento em si, foi composto por dez sessões, com até 90 minutos cada. 0 modelo do programa de treinamento usou procedimentos da análise do comportamento, da aprendizagem social e da teoria cognitiva (BOLSONI-SILVA AT, et al., 2009; CABALLO V, 2009; DEL PRETTE A e DEL PRETTE ZAP, 2011b). As técnicas utilizadas foram modelação, reforçamento, instrução, role-playing e ensaio comportamental.

Para avaliação da efetividade do treinamento em HS foram aplicados os seguintes instrumentos de coleta de dados antes e depois do treinamento: questionário sociodemográfico; Inventário de Habilidades Sociais (IHS-Del-Prette) e o a versão brasileira do questionário de qualidade de vida SF-36.

O questionário sociodemográfico foi composto por nove questões sobre idade, sexo, nível de escolaridade e tempo de vínculo empregatício. O Inventário de Habilidades Sociais (IHS-Del-Prette) é composto por 38 itens que descrevem situações de interação social em diferentes contextos da vida dos sujeitos, como trabalho, família, lazer. O questionário de qualidade de vida SF-36 é formado por 36 itens, englobados em oito escalas (DEL PRETTE A e DEL PRETTE ZAP, 2001).

Para análise dos dados do questionário sócio demográfico, foi utilizado o teste de Igualdade de duas proporções para caracterizar a distribuição da frequência relativa (percentuais) em suas variáveis qualitativas. Para a análise dos dados no IHS-Del-Prette, foi realizada a comparação entre os escores totais de cada fator, pré-intervenção e pós-intervenção.

REAS/EJCH | Vol.12(10) | e4038 | DOI: https://doi.org/10.25248/reas.e4038.2020 Página 3 de 8 
Para tratamento estatístico para IHS (pré-intervenção e pós-intervenção), se utilizou Teste de Wilcoxon (CONOVER WJ, 1971). Na versão brasileira do questionário de qualidade de vida SF-36, se considera valores superiores a 70 como estado positivo de saúde. Para correlacionar os dois instrumentos SF-36 e IHS-DelPrette se usou a Correlação de Spearman (FONSECA BC, et al., 1996). Esta pesquisa recebeu aprovação do Comitê de Ética e Pesquisa, via Plataforma Brasil com número do parecer 1.753.248.

\section{RESULTADOS}

Do total dos 10 profissionais que participaram do estudo $80 \%$ eram mulheres e $20 \%$ homens, com faixa etária média de 27,5 anos. Destes profissionais 50\% moravam sozinhos, $20 \%$ com o cônjuge, $20 \%$ residia com outros parentes e 10\% com pais. Quanto ao tempo de vínculo empregatício $90 \%$ tinham menos de 5 anos na empresa, sendo $10 \%$ mais de 15 anos na empresa. A maioria dos profissionais possuía renda entre 2 a 3 salários mínimos (50\%), sendo que $30 \%$ recebia até 5 salários mínimos e $20 \%$ entre 5 a 10 salários mínimos.

Para saber se o treinamento surtiu efeito e se teve significância estatística, foi usado o teste de Wilcoxon. O Gráfico 1 mostra os resultados em relação escore fatorial, a partir dos dados normativos do estudo original do IHS-Del-Prette e calculado em termos de percentis (DEL PRETTE A e DEL PRETTE ZAP, 2001).

Gráfico 1 - Resultados dos Escores de IHS Pré-intervenção e Pós-intervenção.

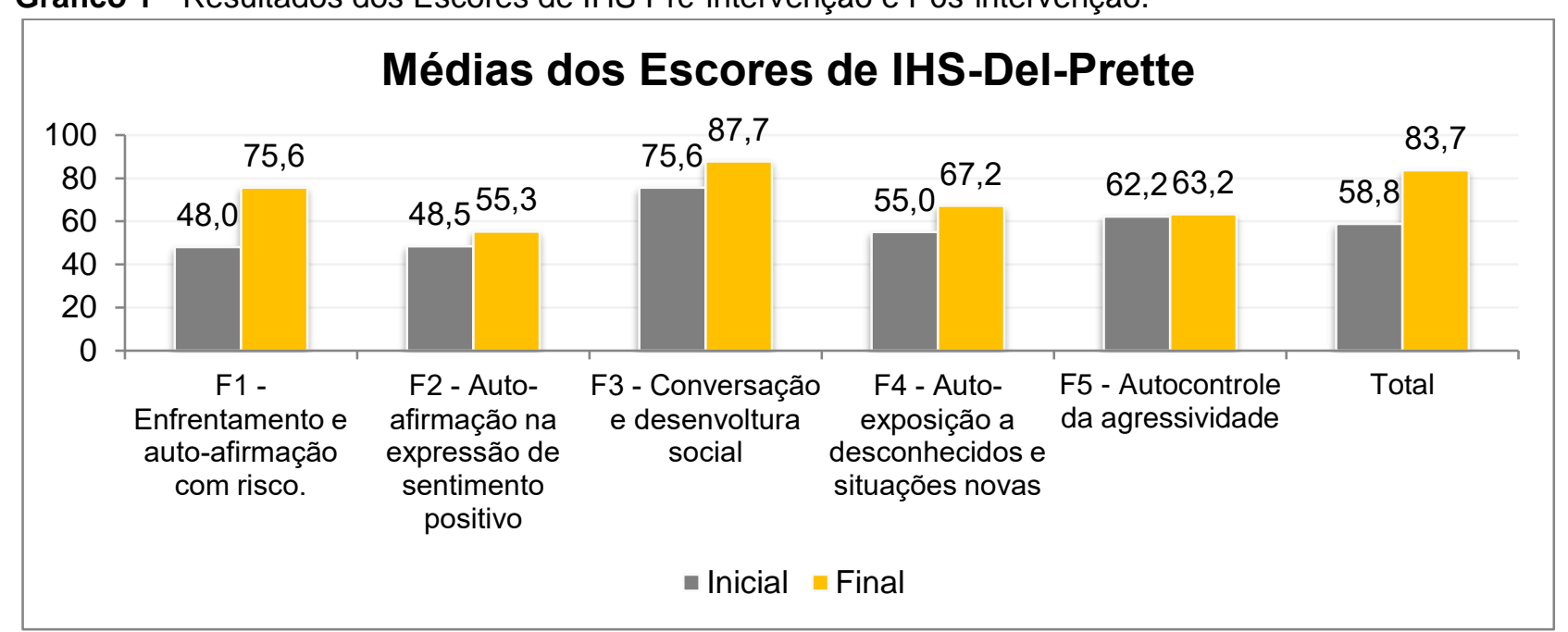

Fonte: Branco PR e Andrade EAA, 2020.

Conforme Gráfico 1 foi possível notar um aumento nas médias dos escores totais dos fatores pósintervenção (final) em relação a pré-intervenção (inicial). No fator F1 - Enfrentamento e Autoafirmação com risco a média antes do THS foi de 48,0 passando para 75,6 na avaliação final. No F2 - Autoafirmação na expressão de sentimento positivo a média dos participantes foi de 48,5 passando 55,3 . O fator que apresentou maior pontuação, em ambas fases (inicial e final) foi o F3 - Conversação e desenvoltura social. Para o F5 Autocontrole da agressividade as médias de 62,2 para 63,2 na pós-intervenção. Em relação ao total das médias foram de 58,8 para 83,7. As médias são usadas como estatística descritiva para entender o que ocorreu nos resultados.

O resultado total (média total de todos os fatores) mostra que a média antes do treinamento foi de $58,8 \mathrm{e}$ passou para 83,7 ( $\mathrm{p}$ valor $=0,005$ ) depois do treinamento, mostrando significância estatística pelo teste de Wilcoxon, ou seja, o treinamento em habilidades sociais para profissionais de atendimento ao cliente surte efeito (há diferença entre antes e depois).

Os participantes do THS foram avaliados com o instrumento SF-36, com o objetivo de avaliar a qualidade de vida de vida dos profissionais. Os domínios deste inventário são: Capacidade Funcional; Aspectos físicos, Percepção de dor, Estado Geral de Saúde, Vitalidade, Aspectos Sociais, Aspectos emocionais e Saúde Mental. Os "scores" em cada domínio variam entre zero e 100, com o zero representando o pior resultado e 100 um estado de saúde perfeito (CICONELLI RM, et al., 1999). 
Através da correlação de Spermean, foi realizada a conexão entre os dois instrumentos aplicados. Foi possível observar que domínios semelhantes de instrumentos diferentes se correlacionaram positivamente, resultando em uma correlação boa e ótima. O domínio Aspecto Social (SF-36), tem entre seus itens a integração de vivências sociais, e o F1 - Enfrentamento e Autoafirmação com risco (IHS) apresenta itens de desenvoltura social, principalmente na apresentação de comportamentos assertivos, e o F3- Conversação e desenvoltura social aborda as vivências sociais. Os resultados mostraram mais significância estatística no momento pós-intervenção, onde os participantes já haviam se submetido ao THS para atendimento ao cliente (Tabela 1).

Tabela 1 - Caracterização da correlação ente IHS-Del-Prette e SF-36 dos trabalhadores n=10, São Paulo SP, 2020.

\begin{tabular}{|c|c|c|c|c|c|c|c|c|c|}
\hline & & $\begin{array}{l}\text { Capac. } \\
\text { Func. }\end{array}$ & $\begin{array}{l}\text { Aspec. } \\
\text { Físic. }\end{array}$ & Dor & $\begin{array}{l}\text { Geral } \\
\text { Saúde }\end{array}$ & Vital & $\begin{array}{l}\text { Asp. } \\
\text { Sociais }\end{array}$ & $\begin{array}{l}\text { Asp. } \\
\text { Emoc. }\end{array}$ & $\begin{array}{l}\text { Saúde } \\
\text { Mental }\end{array}$ \\
\hline \multicolumn{10}{|c|}{ Inicial } \\
\hline \multirow{2}{*}{$\mathrm{F} 1$} & Corr(r) & $-23,70 \%$ & $-21,10 \%$ & $-29,00 \%$ & $3,70 \%$ & $-30,00 \%$ & $-64,10 \%$ & $-26,80 \%$ & $-49,20 \%$ \\
\hline & Pvalor & 0,51 & 0,559 & 0,416 & 0,919 & 0,399 & 0,046 & 0,455 & 0,148 \\
\hline \multirow{2}{*}{ F2 } & Corr(r) & $-45,10 \%$ & $-1,00 \%$ & $-33,60 \%$ & $-31,10 \%$ & $4,90 \%$ & $-30,40 \%$ & $-6,90 \%$ & $-34,40 \%$ \\
\hline & Pvalor & 0,191 & 0,978 & 0,342 & 0,382 & 0,893 & 0,394 & 0,85 & 0,331 \\
\hline \multirow{2}{*}{ F3 } & Corr(r) & $1,20 \%$ & $-4,40 \%$ & $-8,00 \%$ & $8,50 \%$ & $59,80 \%$ & $-8,40 \%$ & $-3,90 \%$ & $6,70 \%$ \\
\hline & Pvalor & 0,973 & 0,904 & 0,827 & 0,815 & 0,068 & 0,818 & 0,914 & 0,853 \\
\hline \multirow{2}{*}{ F4 } & Corr $(r)$ & $41,80 \%$ & $27,60 \%$ & $44,20 \%$ & $-8,50 \%$ & $-9,80 \%$ & $12,90 \%$ & $28,10 \%$ & $32,40 \%$ \\
\hline & Pvalor & 0,229 & 0,441 & 0,201 & 0,815 & 0,788 & 0,723 & 0,432 & 0,361 \\
\hline \multirow{2}{*}{ F5 } & Corr(r) & $-25,30 \%$ & $-29,00 \%$ & $-18,00 \%$ & $19,50 \%$ & $-6,10 \%$ & $-4,20 \%$ & $-36,00 \%$ & $-40,20 \%$ \\
\hline & Pvalor & 0,48 & 0,417 & 0,618 & 0,589 & 0,866 & 0,909 & 0,307 & 0,25 \\
\hline \multirow{2}{*}{ Total } & Corr $(r)$ & $3,10 \%$ & $6,70 \%$ & $-12,20 \%$ & $23,60 \%$ & $-26,20 \%$ & $-36,10 \%$ & $3,30 \%$ & $-34,10 \%$ \\
\hline & Pvalor & 0,933 & 0,854 & 0,738 & 0,511 & 0,464 & 0,306 & 0,929 & 0,334 \\
\hline \multicolumn{10}{|c|}{ Final } \\
\hline \multirow{2}{*}{$\mathrm{F} 1$} & Corr(r) & $-28,40 \%$ & $-4,00 \%$ & $-33,60 \%$ & $-38,40 \%$ & $-27,90 \%$ & $-64,90 \%$ & $-14,70 \%$ & $-38,70 \%$ \\
\hline & Pvalor & 0,427 & 0,912 & 0,342 & 0,273 & 0,435 & 0,042 & 0,685 & 0,27 \\
\hline \multirow{2}{*}{ F2 } & Corr(r) & $-45,70 \%$ & $-12,10 \%$ & $-39,10 \%$ & $-22,60 \%$ & $-20,90 \%$ & $-50,60 \%$ & $-18,70 \%$ & $-48,80 \%$ \\
\hline & Pvalor & 0,184 & 0,738 & 0,263 & 0,531 & 0,563 & 0,136 & 0,606 & 0,153 \\
\hline \multirow{2}{*}{ F3 } & Corr(r) & $-37,20 \%$ & $-37,50 \%$ & $-25,80 \%$ & $-28,70 \%$ & $-44,60 \%$ & $-85,00 \%$ & $-45,00 \%$ & $-57,20 \%$ \\
\hline & Pvalor & 0,29 & 0,285 & 0,472 & 0,421 & 0,196 & 0,002 & 0,192 & 0,084 \\
\hline \multirow{2}{*}{ F4 } & Corr $(r)$ & $-24,70 \%$ & $21,90 \%$ & $-21,40 \%$ & $-16,50 \%$ & $-4,30 \%$ & $-18,80 \%$ & $15,10 \%$ & $-26,10 \%$ \\
\hline & Pvalor & 0,492 & 0,543 & 0,553 & 0,649 & 0,906 & 0,602 & 0,678 & 0,467 \\
\hline \multirow{2}{*}{ F5 } & Corr $(r)$ & $-19,10 \%$ & $-20,90 \%$ & $-21,70 \%$ & $-11,60 \%$ & $-55,80 \%$ & $-52,30 \%$ & $-28,80 \%$ & $-46,90 \%$ \\
\hline & Pvalor & 0,596 & 0,562 & 0,547 & 0,75 & 0,093 & 0,121 & 0,419 & 0,171 \\
\hline \multirow{2}{*}{ Total } & Corr(r) & $-16,00 \%$ & $7,10 \%$ & $-27,20 \%$ & $-18,30 \%$ & $-30,10 \%$ & $-54,40 \%$ & $-2,30 \%$ & $-38,30 \%$ \\
\hline & Pvalor & 0,658 & 0,846 & 0,447 & 0,613 & 0,399 & 0,104 & 0,95 & 0,274 \\
\hline
\end{tabular}

Legenda: F1 - Enfrentamento e auto-afirmação com risco; F2- Auto-afirmação na expressão de sentimento positivo; F3 - Conversação e desenvoltura social; F4 - Auto-exposição a desconhecidos e situações novas.

Fonte: Branco PR e Andrade EAA, 2020.

Os resultados mostram que existem duas correlações estatisticamente significantes: o escore do Aspecto Social do SF-36 com Fator 1 - Enfrentamento e Autoafirmação com risco, com valor de $64,1 \%$ com ( $p=0,046)$ na pré-intervenção (inicial) e -64,9\% ( $p-0,042)$ na pós-intervenção (final) e com o Fator 3 - Conversação e desenvoltura social, com -85,0\% (p-0,002) no momento final. Essas correlações são negativas o que indicam que quanto maior o escore de Aspecto Social, menor o valor do escores desses dois fatores. Essas correlações são classificadas como sendo Boa e Ótima. 
No domínio Vitalidade, na fase pré-intervenção (inicial) 59,8\% com o F3 - Conversação e desenvoltura social $(p-0,068)$ que por estarem próximos do limite de aceitação, são considerados que tendem a ser significativos. Na pós-intervenção também houve aproximação de significância do domínio Vitalidade (final) $55,8 \%$ (p-0,093) com o F5- Autocontrole e Agressividade. E no domínio Saúde mental 57,2\% (p-0,084) houve uma aproximação de correlação Fator 3 - Conversação e desenvoltura social. Assim, a correlação entre IHSDel-Prette e SF-36, mostra que ambos instrumentos tem uma correlação positiva entre Aspecto Social (SF36) com Fator 1 - Enfrentamento e Autoafirmação com risco (IHS), com valor de -64,9\% e com o Fator 3 Conversação e desenvoltura social (IHS).

Entendemos que este THS para o atendimento ao cliente, se propôs a cumprir os objetivos propostos. Pode-se observar que domínios semelhantes de instrumentos diferentes se correlacionaram positivamente, resultando em uma correlação boa e ótima. Há significância estatística no momento pós-intervenção, onde os participantes já haviam se submetido ao THS para atendimento ao cliente.

\section{DISCUSSÃO}

Os resultados apresentados mostram que houve aumento nas médias do IHS-Del-Prette, com relevância estatística. Os participantes ampliaram seus repertórios em HS, ofertando mais qualidade de vida para os trabalhadores, potencializando suas capacidades de lidar com estresses do trabalho, melhorando suas relações interpessoais, contribuindo para um funcionamento social positivo. Dentro de uma perspectiva de promoção a saúde, com foco na saúde e não da doença, podemos defender que este estudo se harmoniza com tantos outros que mostram a importância de HS como fator de proteção e prevenção (MURTA SG, 2005; BOLSONI AT, et al., 2008; ABREU S, et al., 2016; DEL PRETTE A et al., 2015).

Em uma revisão da literatura sobre programas de prevenção em saúde mental no Brasil, Abreu $S$, et al. (2016) identificaram 25 artigos que relatavam 42 tipos de intervenção. As Habilidades $S$ representavam "41,19\%" da frequência total das intervenções promovidas. Quanto às características dos estudos, nota-se a predominância de intervenções em grupo, de longa duração, baseadas no modelo teórico comportamental e cognitivo-comportamental, focadas na promoção de HS. Um dos problemas apontados é que estes estudos não se denominavam como preventivos.

A literatura tem sugerido a associação de déficits em habilidade sociais a quadros fobia social, ao consumo de maconha, de bebidas alcoólicas e ao início do uso de tabaco (CABALLO V, 2009; WAGNER MF e OLIVEIRA MSN, 2009). LessaHorta R, et al. (2016), em um estudo com uma amostra conveniência 519 sujeitos usuários de crack, revela que $52,8 \%$ dos entrevistados possuíam escores deficitários em HS. Neste estudo se mostrou a importância de incluir a avaliação em HS nos programas de atendimento ao usuário de crack, e que o maior repertório de HS amplia a rede de apoio, aspecto importante no tratamento desses usuários. Com isso, a promoção de HS em mostras não clínicas podem ser uma estratégia de promoção a saúde, focando a psicoeducação.

Outro estudo mostra que naqueles os indivíduos que apresentem maior/melhor reportório de habilidades sociais, essas habilidades podem funcionar como promotoras de gratificações positivas recebidas por parte dos outros Ao permitirem uma interação mais adequada com os que o rodeiam, as habilidades sociais acabam por ter impacto nas relações que o indivíduo estabelece e no modo como este se comporta na relação com os pares (PEIXOTO AC, et al., 2018). Um estudo mostra a influência do ambiente de trabalho sobre a qualidade de vida dos profissionais, por isso consideram importante ofertar aperfeiçoamento laboral com a implementação de medidas que estimulem nos trabalhadores a compreensão da realidade para o próprio benefício e consequente qualidade de vida (DE OLIVEIRA SP, 2020).

Em outra pesquisa sobre a Síndrome de Burnout, em que os autores discutirem o adoecimento causado pelo trabalho, reforçam a importância da construção de estratégias de educação continuada que aliviem frustrações e tensões assim como estimulem a motivação dos trabalhadores, criando cenários de diálogos entre os envolvidos. Os mesmos autores reforçam que intervenções preventivas devem ser implementadas de acordo com a necessidades dos profissionais e devem ser considerados recursos multifatoriais, que influenciam no quadro de adoecimento (DA SILVA JF, et al, 2020). 
A criação de espaços de discussões coletivas são fundamentais para o fortalecimento da autonomia e diminuição de sofrimento mental entre trabalhadores, neste sentido, os autores sugerem que as organizações de trabalho propiciem a convivência, possibilitem então o bem viver em sociedade, possibilitando a construção de subjetividades saudáveis na equipe (SALDANHA DA SILVEIDA CC, et al., 2019)

Os resultados do presente estudo demonstram que o THS é uma estratégia em promoção a saúde, pois há evidências que fatores de risco para a saúde mental são habilidades sociais pobres, estilo de enfretamento negativo, baixa autoestima, baixa eficácia, entre outros. Já entre os fatores de proteção a saúde mental, está intimamente ligado a um repertório diversificado de HS, autoestima, educação, práticas parentais saudáveis (ABREU S, et al., 2016, DEL PRETTE ZAP, et al., 2015). Del Prette ZAP, et al. (2015), mostram o THS é uma intervenção em saúde mental e que ter um bom repertório está ligado ao sucesso profissional.

Muitos avanços em relação a saúde do trabalhador foram conquistados ao longo das últimas décadas. Especialmente no Brasil há uma política de saúde do trabalhador que oferta um espoco de ações para a massa producente e tem como um dos objetivos a melhoria da qualidade de vida, a realização pessoal e social dos trabalhadores, sem gerar prejuízo para sua saúde, integridade física e mental (MATTOS RCOC, et al., 2019). Entretanto, é sabido que ainda faltam programas preventivos e de promoção da saúde que tenham a função de proteger a saúde mental destes trabalhadores.

Os programas de THS para o ambiente de trabalho podem contribuir para o fortalecimento do sujeito em suas atividades, ampliando seu repertório e ofertando diversas soluções para os desafios sociais do trabalho, sendo estes ganhos passíveis de generalização, se estendendo outras esferas da vida. Com este cenário, vemos uma intervenção de promoção a saúde, e consequente empoderamento dos indivíduos pois ampliaram o repertório de habilidades sociais, através das técnicas de exposição dialogada da modelação, a significância das HS aprendidas e o aumento das médias depois da intervenção.

A saúde do trabalhador é influenciada por diversos fatores sociais, econômicos, políticos, biológicos e psicológicos (FONSECA BC, et al., 2019). Coube aqui neste estudo focalizar um desses aspectos, o que não diminui a importância de todos os outros. Conforme revisão da literatura as HS são comportamentos sociais requeridos ao longo da vida. Ter HS contribui para a qualidade de vida, além de ser um fator de proteção em saúde mental. Compete aqui considerar uma limitação deste estudo: o tamanho da amostra, considerada pequena, o que não nos permite fazer generalizações. Sugere-se que próximos estudos possam comtemplar estas questões.

\section{CONCLUSÃO}

Os resultados deste trabalho mostraram que o Treinamento em Habilidades Sociais ampliou o repertório de habilidades sociais dos trabalhadores envolvidos, pois potencializou a capacidade de lidar com estresses, melhorou as relações interpessoais e contribuiu para um funcionamento social positivo. Como os fatores de proteção à saúde mental, estão intimamente ligados à um repertório diversificado de Habilidades Sociais, conclui-se que o treinamento pode ser considerado uma estratégia efetiva de promoção à saúde, pois repercute positivamente na construção de um ambiente saudável para os relacionamentos, melhorando 0 bem estar físico, mental e social, contribuindo então para a qualidade de vida dos trabalhadores e do contexto de trabalho.

\section{REFERÊNCIAS}

1. ABREU S, et al. Programas preventivos brasileiros: quem faz e como é feita a prevenção em saúde mental? PsicoUSF, 2016; 21(1): 163-177.

2. ANDRADE EA, BOGUS CM. Políticas públicas dirigidas à juventude e promoção da saúde: como a proposta de auxiliares da juventude foi traduzida em prática. Interface (Botucatu), 2010; 14(35): 853-866.

3. BOLSONI-SILVA AT, et al. Promovendo habilidades sociais educativas parentais na prevenção de problemas de comportamento. Revista Brasileira de Terapia Comportamental e Cognitiva, 2008; 10(2): 125-142.

4. BOLSONI-SILVA AT; et al. Avaliação de um Treinamento de Habilidades Sociais (THS) com Universitários e RecémFormados. Interação em Psicologia, Curitiba, 2009; 13(2): 241-251.

5. DA SILVA JF, et al. Síndrome de Burnout em profissionais de Enfermagem no contexto da Atenção Básica. Revista Eletrônica Acervo Saúde, 2020, 39 (e2320). 
6. DE OLIVEIRA SP, et al. Qualidade de vida no trabalho de manipuladores de um serviço de nutrição hospitalar em Maceió - AL. Revista Eletrônica Acervo Saúde, 2020, 50 (e3498).

7. BRASIL. Portaria no 1.823, de 23 de agosto de 2012. Institui a Política Nacional de Saúde do Trabalhador e da Trabalhadora. Diário Oficial da União, 2012.

8. CABALLO VE, et al. Abordagem cognitiva na avaliação e intervenção sobre habilidades sociais. In: DEL PRETTE ZAP, DEL PRETTE A. (Orgs). Psicologia das Habilidades Sociais: diversidade teórica e suas implicações. Editora Vozes, 2009.

9. CAMPOS JR, et al. Relações entre depressão, habilidades sociais, sexo e nível socioeconômico em grandes amostras de adolescentes. Psicologia Teoria e Pesquisa ,2018; 34(e3446).

10. CICONELLI RM, et al. Tradução para a língua portuguesa e validação do questionário genérico de avaliação de qualidade de vida SF-36 (Brasil SF-36). Revista Brasileira Reumatologia, 1999;39(3):143-50.

11. CONOVER WJ. Practical nonparametric statistics, New York; 1971.

12. DEL PRETTE A, DEL PRETTE ZAP. Psicologia das relações interpessoais: Vivências para um trabalho em grupo. Editora Vozes, Petrópolis, 2001; 232p.

13. DEL PRETTE A, DEL PRETTE ZAP. Inventário de habilidades sociais (IHS-DelPrette): manual de aplicação, apuração e interpretação [Social Skills Inventory (IHS-DelPrette): user's guide for use, data processing and interpretation]. Casa do psicólogo, São Paulo, 2001.

14. DEL PRETTE A, DEL PRETTE ZAP. No contexto da travessia para o ambiente de trabalho: treinamento de habilidades sociais com universitários. Estudos de Psicologia, 2003; 8(3):413-420.

15. DEL PRETTE A, DEL PRETTE ZAP. Programa vivencial de habilidades sociais: Características sob a perspectiva da análise do comportamento. In: GARCIA, M.R.; ABREU, P.R.; CILLO, E.N.P.; FALEIROS, P.B.; QUEIROZ, P.P. (Org.). Comportamento e Cognição: Terapia Comportamental e Cognitiva, Santo André, Esetec. 2010; 187-229

16. DEL PRETTE A, DEL PRETTE ZAP. Psicologia das relações interpessoais: Vivências para um trabalho em grupo. Petrópolis, Editora Vozes, 2011; 231p.

17. DEL PRETTE ZAP, et al. Habilidades sociais ao longo do desenvolvimento: perspectivas de intervenção em saúde mental. In: MURTA SG et al. (Orgs). Prevenção e Promoção em saúde Mental - Fundamentos, Planejamento e Estratégias. Novo Hamburgo, 2015; Sinopsy.

18. FALCON EMO. A avaliação de um programa de treinamento da empatia com universitários. Revista Brasileira de Terapia Comportamental e Cognitiva, 1999; São Paulo, 1: 23-32.

19. FONSECA BC, et al. Planejamento de intervenções em Saúde do Trabalhador no território: uma experiência participativa. Revista Brasileira de Saúde Ocupacional, 2019; 44:(36), 1-9.

20. IBGE (Instituto Brasileiro de Geografia, Estatística). Informativos econômicos da Secretaria de Política Econômica (SPE) PNADc IBGE referente trimestre set-nov/19, 2020. Disponível em: http://www.fazenda.gov.br/centrais-deconteudos/publicacoes/conjuntura-economica/emprego-e-renda/2019/ie-pnadc-novembro-2019.pdf.

21. LESSAHORTA R, et al. Condições associadas a prejuízo de desempenho em habilidades sociais em uma amostra de conveniência de usuários de crack. Cadernos de Saúde Pública, 2016; 32: e00010715.

22. MATTOS RCOC, et al. Formação profissional como ação estratégica para implementação da Política Nacional de Saúde do Trabalhador e da Trabalhadora. Rev. bras. saúde ocup., São Paulo, 2019, 44 (e24).

23. MURTA SG. Aplicações do Treinamento em Habilidades Sociais: Analise da Produção Nacional. Psicologia Reflexão e Critica, 2005; 18(2): 283-291.

24. O.M.S. Organização Ambientes de trabalho saudáveis: um modelo para ação: para empregadores, trabalhadores, formuladores de política e profissionais. /OMS; tradução do Serviço Social da Indústria. - Brasília: SESI/DN,26 p.: il. 2010

25. PEIXOTO AC, et al. Habilidades sociais na promoção de saúde: preditoras da saúde mental e sexual. Psicologia, Saúde \& Doenças, Lisboa, 2018; 19(1):11-17.

26. RONDINA RC. A relação entre tabagismo e habilidades sociais: uma revisão da literatura. Revista Brasileira de Terapia Cognitivo comportamental, 2010; 6(2): 80-93.

27. SADIR MA, LIPP MN. Influência do treino de controle do estresse nas relações interpessoais no trabalho. O Mundo da Saúde, 2013; 37(2):131-140.

28. SALDANHA DA SILVEIDA CC, et al. Sofrimento de trabalhadores da atenção básica em um contexto de tragédia: estratégias para evitar o adoecimento. Revista Eletrônica Acervo Saúde, 2019, 11(15): e1296.

29. SCHIRRMEISTER R, LIMONGI-FRANÇA C. A Qualidade de Vida no Trabalho: Relações com o Comprometimento Organizacional nas Equipes Multicontratuais. Revista Psicologia: Organizações e Trabalho, Brasília, 2012; 12(3):283298.

30. SEGRIN C, FLORA J. Poor social skills are a vulnerability fator in the development of psychosocial problems. Human Communication Research, 2000; 26(3):489-514.

31. WAGNER, MF, OLIVEIRA, MSN. Estudo das habilidades sociais em adolescentes usuários de maconha. Psicologia em Estudo, Maringá, 2009; 14(1):101-110.

32. W.H.O., Life skills education in schools. Geneva, 1997. 